\title{
On the univalence of an integral on a subclass of meromorphic convex univalent functions
}

\author{
I. R. Nezhmetdinov and S. Ponnusamy
}

(Received February 18, 2002)

\begin{abstract}
A nonlinear integral operator is studied on the class of convex meromorphic functions in the exterior of the unit disk. In this paper, we improve a sufficient condition for univalency of the operator obtained earlier by the first author.
\end{abstract}

Key words: univalent, meromorphic, convex functions and integral operators.

\section{Introduction and main results}

Let $\mathcal{S}$ be the class of normalized functions $f(z)=z+a_{2} z^{2} \ldots$, analytic and univalent in the unit disk $E=\{z \in \mathbb{C}:|z|<1\}$. In [5] an integral operator $P_{\lambda}[f]$ defined by

$$
P_{\lambda}[f](z)=\int_{0}^{z}\left(f^{\prime}(t)\right)^{\lambda} d t
$$

was shown to map $\mathcal{S}$ into itself, provided that $|\lambda| \leq(\sqrt{5}-2) / 3=0.078 \ldots$ Becker [3] established the univalence of $P_{\lambda}[f]$ for $|\lambda| \leq 1 / 6$ whereas Royster [10] gave an example implying that, unless $\lambda=1$, for any $\lambda$ outside of the disk $|\lambda| \leq 1 / 3$ a function $f_{0} \in \mathcal{S}$ can be found such that $P_{\lambda}\left[f_{0}\right] \notin \mathcal{S}$. Pfaltzgraff [8] improved the range of $\lambda$ to $|\lambda| \leq 1 / 4$. The question, whether the operator $P_{\lambda}$ preserves univalency for $1 / 4<|\lambda| \leq 1 / 3$ still remains open.

A similar problem is completely solved for the subclass $\mathcal{K} \subsetneq \mathcal{S}$ of univalent convex functions. Namely, the inclusion $P_{\lambda}[\mathcal{K}] \subset \mathcal{S}$ holds if and only if either $|\lambda| \leq 1 / 2$ or $\lambda$ is real with $1 / 2 \leq \lambda \leq 3 / 2$ (see $[2,8]$ ). More results of the similar type for other subclasses of $\mathcal{S}$ are obtained in $[6,9]$.

Counterparts of these problems for the case of meromorphic functions were studied by a number of authors (see $[1,2,11]$ ), however, the relevant constants are smaller than in the regular case. Denote by $\Sigma$ be the class of function

$$
f(\zeta)=\zeta+\sum_{k=0}^{\infty} \alpha_{k} \zeta^{-k},
$$


regular and univalent in $E^{-}=\{\zeta: 1<|\zeta|<\infty\}$ and having a simple pole at $\zeta=\infty$. Let $\Sigma_{\mathcal{K}}$ be its subclass consisting of convex univalent functions. Define the following integral operator

$$
P_{\lambda}[f](\zeta)=\int_{\zeta_{0}}^{\zeta}\left(f^{\prime}(t)\right)^{\lambda} d t
$$

with $\lambda \in \mathbb{C}, \zeta_{0} \in E^{-}$.

Recently the first author [7] applied a condition for univalency by J. Becker [4] to show that $P_{\lambda}[\Sigma] \subset \Sigma$ for all $|\lambda| \leq 1 / 4$. The following result regarding the set

$$
\Lambda\left(\Sigma_{\mathcal{K}}, \Sigma\right)=\left\{\lambda \in \mathbb{C}: P_{\lambda}\left[\Sigma_{\mathcal{K}}\right] \subset \Sigma\right\}
$$

was established in [7].

Theorem 1.2 We have

$$
\left\{\lambda=\mu e^{i \nu}:|\lambda| \leq \tilde{\mu}_{0}(\nu)\right\} \subset \Lambda\left(\Sigma_{\mathcal{K}}, \Sigma\right),
$$

with

$$
\tilde{\mu}_{0}(\nu)= \begin{cases}(3+\cos \nu) / 4 & \text { for } \nu_{0} \leq|\nu| \leq \pi, \\ (1+|\sin \nu|)^{-1} & \text { for } 0 \leq|\nu| \leq \nu_{0} .\end{cases}
$$

Here $\nu_{0}=\arccos t_{0}=0.9633 \ldots$, where $t_{0}=0.5707 \ldots$ is the unique positive real root of the equation

$$
47-52 t-46 t^{2}-12 t^{3}-t^{4}=0
$$

in the interval $(0,1)$.

The function $\tilde{\mu}_{0}(\nu)$ appears to have a jump at $\nu=\nu_{0}$ which is only due to the method of proof. In the present paper we improve the result by removing the discontinuity. However, the second expression for $\tilde{\mu}_{0}(\nu)$ is unsuperseded albeit in a small neighborhood of $\nu=0$. Our main result is as follows.

Theorem 1.3 Given any real $\nu$ such that $|\nu| \leq \nu_{0}$, where $\nu_{0}$ is defined in the previous theorem, we have $\left\{\lambda=\mu e^{i \nu}:|\lambda| \leq \mu_{0}(\nu)\right\} \subset \Lambda\left(\Sigma_{\mathcal{K}}, \Sigma\right)$, with

$$
\mu_{0}(\nu)=\frac{2 \cos \theta_{\nu}-1-\cos \nu}{2\left(1-\cos \left(\nu-\theta_{\nu}\right)\right)}
$$


$\theta_{\nu}$ being the unique positive real root of the equation

$$
\begin{aligned}
F(\theta):= & 7-5 \cos ^{2} \nu+\left(17-6 \cos \nu-11 \cos ^{2} \nu\right) \cos \theta \\
& \quad-\left(4+10 \cos \nu-8 \cos ^{2} \nu\right) \cos ^{2} \theta-\left(12-16 \cos ^{2} \nu\right) \cos ^{3} \theta \\
- & (8+14 \cos \theta) \sin \nu \sin \theta-\left(1-4 \cos \theta-8 \cos ^{2} \theta\right) \sin 2 \nu \sin \theta \\
= & 0
\end{aligned}
$$

in the interval $0<\theta<\theta_{\nu}^{\prime}=2 \arctan [\sin \nu /(3+\cos \nu)]$.

Proof. By reasoning as in [7] we can conclude that the inclusion $P_{\lambda}\left[\Sigma_{\mathcal{K}}\right] \subset$ $\Sigma$ holds for a given $\lambda=\mu e^{i \nu}$ if there exists some $c,|c| \leq 1$, such that

$$
\begin{aligned}
H(\rho, \lambda, c) & =\frac{2 \mu|\rho-c|}{|1-c|(\rho+1)}+\frac{1}{\rho}\left|\frac{2 \lambda(\rho-c)}{(1-c)(\rho+1)}+c\right| \\
& \leq 1 \quad \text { for all } \rho>1 .
\end{aligned}
$$

In particular, we have

$$
H(1, \lambda, c)=\mu+|\lambda+c| \leq 1
$$

and

$$
H(\infty, \lambda, c)=2 \mu /|1-c| \leq 1 .
$$

Assume that the two bounds are attained and we try to determine the largest value of $\mu$ for which (1.6) holds. Set $c=1-2 \mu e^{i \theta}$, whence $|c| \leq 1$ is equivalent to the inequality $\mu \leq \cos \theta$. On the other hand, if the estimate in (1.7) is attained, then we have

$$
\mu=\frac{2 \cos \theta-1-\cos \nu}{2(1-\cos (\nu-\theta))}
$$

Due to the previous studies it suffices to consider the case when $0<$ $\nu<\nu_{0}$ and $|\theta|<\pi / 2$. Since it is of interest only to find $\mu>1 / 2$, we get from (1.9) that

$$
0<\theta<2 \arctan (\sin \nu /(2+\cos \nu)) .
$$

Note that from (1.9) it also follows that $|c| \leq 1$.

By using a standard method of computation, we find that $\mu$ is an increasing function of $\theta$ on $\left[0, \theta_{\nu}^{\prime}\right]$, besides, there is also some numerical evidence that the global maximum for $\mu(\nu)$ is to be found here than on the 
remaining part of the previous interval. So, in what follows we shall confine ourselves to considering of (1.9) on the interval $\left[0, \theta_{\nu}^{\prime}\right]$.

It can be easily seen that (1.6) is equivalent to the following condition

$$
\left|(\rho-c) e^{i(\nu-\theta)}+c(\rho+1)\right| \leq \rho(\rho+1-|\rho-c|), \quad \rho \geq 1,
$$

where the right-hand side is positive if $|c| \leq 1$. Therefore, we can square both sides of the inequality (1.10) without violating it. Thus,

$$
\rho^{2}|\rho-c| \leq \rho^{3}-\rho^{2} \operatorname{Re} c+A_{1} \rho+A_{0}, \quad \rho \geq 1,
$$

where

$$
A_{1}=\operatorname{Re}\left(c-\bar{c} e^{i(\nu-\theta)}\right), \quad A_{0}=-|c|^{2}(1-\cos (\nu-\theta)) .
$$

Clearly, both sides in (1.11) are positive for all $\rho \geq 1$, so we may square it again. After some elementary transformations, we write the resulting inequality as

$$
a_{4} \rho^{4}+a_{3} \rho^{3}+a_{2} \rho^{2}+a_{1} \rho+a_{0} \geq 0, \quad \rho \geq 1,
$$

where the coefficients are defined by the relations

$$
\begin{aligned}
& a_{4}=2 A_{1}-(\operatorname{Im} c)^{2}, \quad a_{3}=-2\left(A_{1} \operatorname{Re} c-A_{0}\right), \\
& a_{2}=-2 A_{0} \operatorname{Re} c+A_{1}^{2}, \quad a_{1}=2 A_{0} A_{1}, \quad a_{0}=A_{0}^{2} .
\end{aligned}
$$

We recall that in [7] it was possible to prove that $a_{4} \geq 0$ for $\theta=\theta_{\nu}^{\prime}$, provided that $\nu_{0} \leq|\nu| \leq \pi$. However, when $0 \leq|\nu|<\nu_{0}, \theta=\theta_{\nu}^{\prime}$, we get $a_{4}<0$, so that the inequality (1.13) fails for sufficiently large $\rho$. Now, let us choose $\theta$ so that $a_{4}=0$, and prove that (1.13) is still valid.

Note that for $\rho=1$ both sides of the inequality (1.10) are equal to $2 \mu$, therefore $\rho=1$ is a root of the polynomial in (1.13). Hence, it suffices to prove that

$$
a_{3} \rho^{2}+\left(a_{3}+a_{2}\right) \rho-a_{0} \geq 0, \quad \rho \geq 1 .
$$

This is implied by the following inequalities

$$
a_{3} \geq 0 \text { and } 2 a_{3}+a_{2}-a_{0} \geq 0 .
$$

Now we proceed to check (1.17). By substituting (1.9) into $c=1-2 \mu e^{i \theta}$ and then into (1.12) we obtain 


$$
\begin{array}{r}
\operatorname{Re} c=\frac{(1-\cos \theta)(1+2 \cos \theta)-\sin \nu \sin \theta}{1-\cos (\nu-\theta)} \\
\begin{array}{r}
A_{1}=\frac{1-\cos \theta}{1-\cos (\nu-\theta)}\left[3+\cos \theta+2\left(1-\cos \theta-2 \cos ^{2} \theta\right) \cos \nu\right. \\
-2(1+2 \cos \theta) \sin \nu \sin \theta]
\end{array}
\end{array}
$$

and

$$
\begin{array}{r}
A_{0}=-\frac{1-\cos \theta}{1-\cos (\nu-\theta)}\left[2+3 \cos \theta+\left(1-2 \cos \theta-4 \cos ^{2} \theta\right) \cos \nu\right. \\
-2(1+2 \cos \theta) \sin \nu \sin \theta] .
\end{array}
$$

After lengthy computations the equation $a_{4}=0$ can be written in an equivalent form as (1.5). Clearly,

$$
F(0)=8(1-\cos \nu)^{2} \geq 0 .
$$

On the other hand, we note that

$$
F\left(\theta_{\nu}^{\prime}\right)=\frac{(1-\cos \nu)^{2}\left(47-52 \cos \nu-46 \cos ^{2} \nu-12 \cos ^{3} \nu-\cos ^{4} \nu\right)}{(5+3 \cos \nu)^{3}}<0,
$$

whenever $0<|\nu|<\nu_{0}$. So, (1.5) has at least one root $\theta_{\nu}$ in the interval $\left(0, \theta_{\nu}^{\prime}\right)$.

To prove the uniqueness of the root, let us verify that $F(\theta)$ is a convex function. We obtain

$$
\begin{aligned}
F^{\prime \prime}(\theta)= & -8-20 \cos \nu+16 \cos ^{2} \nu-\left(89-6 \cos \nu-107 \cos ^{2} \nu\right) \cos \theta \\
& +\left(16+40 \cos \nu-32 \cos ^{2} \nu\right) \cos ^{2} \theta+\left(108-144 \cos ^{2} \nu\right) \cos ^{3} \theta \\
& +(8 \sin \nu+17 \sin 2 \nu) \sin \theta+(56 \sin \nu-16 \sin 2 \nu) \sin \theta \cos \theta \\
& -72 \sin 2 \nu \sin \theta \cos ^{2} \theta
\end{aligned}
$$

By substituting $t=\tan (\theta / 2)$ into this expression we can show that $F^{\prime \prime}(\theta)$ has the same sign as the polynomial

$$
\begin{aligned}
P(t)= & \sum_{j=0}^{6} b_{j} t^{j} \equiv 27+26 \cos \nu-53 \cos ^{2} \nu \\
& +(128 \sin \nu-142 \sin 2 \nu) t-\left(453+94 \cos \nu-619 \cos ^{2} \nu\right) t^{2} \\
& +(32 \sin \nu+356 \sin 2 \nu) t^{3}+\left(373-106 \cos \nu-459 \cos ^{2} \nu\right) t^{4} \\
& -(96 \sin \nu+78 \sin 2 \nu) t^{5}-\left(11-14 \cos \nu+21 \cos ^{2} \nu\right) t^{6},
\end{aligned}
$$


where $0<t<\tau=\sin \nu /(3+\cos \nu)$. Obviously,

$$
b_{0}=(1-\cos \nu)(27+53 \cos \nu)>0 .
$$

Therefore,

$$
b_{0}+b_{1} t \geq t\left(b_{0}+b_{1} \tau\right) / \tau,
$$

and we see that

$$
b_{0}+b_{1} \tau=(1-\cos \nu)\left(209+30 \cos \nu-231 \cos ^{2} \nu\right) /(3+\cos \nu)
$$

is positive. Furthermore, it follows that

$$
b_{0}+b_{1} t+b_{2} t^{2} \geq t^{2}\left(b_{0}+b_{1} \tau+b_{2} \tau^{2}\right) \tau^{-2} .
$$

At the same time, we get

$$
\begin{aligned}
b_{0}+b_{1} \tau+b_{2} \tau^{2}=(1-\cos \nu)(174-248 & \cos \nu-138 \cos ^{2} \nu \\
& \left.+388 \cos ^{3} \nu\right)(3+\cos \nu)^{-2}
\end{aligned}
$$

where the latter expression is positive for $0<|\nu|<\nu_{0}=0.9633 \ldots$

The rest of the proof is done by the same token, just taking into account the following equalities

$$
\begin{gathered}
b_{0}+b_{1} \tau+b_{2} \tau^{2}+b_{3} \tau^{3} \\
=(1-\cos \nu)\left(554+174 \cos \nu+18 \cos ^{2} \nu+282 \cos ^{3} \nu\right. \\
\left.-324 \cos ^{4} \nu\right)(3+\cos \nu)^{-3}, \\
b_{0}+b_{1} \tau+b_{2} \tau^{2}+b_{3} \tau^{3}+b_{4} \tau^{4} \quad+134 \cos ^{2} \nu+138 \cos ^{3} \nu \\
=(1-\cos \nu)\left(2035+1343 \cos \nu-7 \cos ^{4} \nu+135 \cos ^{5} \nu\right)(3+\cos \nu)^{-4}, \\
\quad-125 \\
b_{0}+b_{1} \tau+b_{2} \tau^{2}+b_{3} \tau^{3}+b_{4} \tau^{4}+b_{5} \tau^{5} \\
=(1-\cos \nu)\left(6009+5812 \cos \nu-751 \cos ^{2} \nu+208 \cos ^{3} \nu\right. \\
\left.\quad-21 \cos ^{4} \nu+28 \cos ^{5} \nu-21 \cos ^{6} \nu\right)(3+\cos \nu)^{-5} \\
b_{0}+b_{1} \tau+b_{2} \tau^{2}+b_{3} \tau^{3}+b_{4} \tau^{4}+b_{5} \tau^{5}+b_{6} \tau^{6} \\
=\left(18016+23448 \cos \nu+3616 \cos ^{2} \nu-112 \cos ^{3} \nu+64 \cos { }^{4} \nu\right. \\
\left.\quad+24 \cos ^{5} \nu\right)\left(1-\cos ^{4} \nu\right)(3+\cos \nu)^{-6} .
\end{gathered}
$$


So, for any $\nu, 0<|\nu|<\nu_{0}$, there exists a unique $\theta_{\nu}, 0<\theta_{\nu}<\theta_{\nu}^{\prime}$, such that $F\left(\theta_{\nu}\right)=0$. Equivalently, there exists a unique $d_{\nu}, 3<d_{\nu}<\infty$, such that

$$
\theta_{\nu}=2 \arctan \left[\sin \nu /\left(d_{\nu}+\cos \nu\right)\right]
$$

However, to prove the required inequalities we need to further specify the location of $d_{\nu}$. To this end, let us substitute $\theta=2 \arctan (\sin \nu /(d+$ $\cos \nu)$ ) in (1.5) and write this equation in the equivalent form

$$
\begin{aligned}
p(d, \cos \nu)= & -4(d-2)^{2} \cos ^{4} \nu-\left(12 d^{3}-40 d^{2}+20 d+24\right) \cos ^{3} \nu \\
& -\left(9 d^{4}-12 d^{3}-50 d^{2}+76 d+1\right) \cos ^{2} \nu \\
& +\left(2 d^{5}-30 d^{4}+88 d^{3}-68 d^{2}-10 d+2\right) \cos \nu \\
& +\left(4 d^{6}-22 d^{5}+39 d^{4}-16 d^{3}-14 d^{2}+6 d-1\right) \\
= & 0
\end{aligned}
$$

where $p(d, x)$ is a polynomial with respect to both $d$ and $x$.

Now, it is possible to verify the required estimates. From (1.14)-(1.20) we get

$$
\begin{aligned}
{[1} & -\cos (\nu-\theta)]^{2}(1-\cos \theta)^{-1} a_{3} \\
= & -18-6 \cos \nu+8 \cos ^{2} \nu+\left(-28+12 \cos \nu+20 \cos ^{2} \nu\right) \cos \theta \\
& +\left(10+26 \cos \nu-12 \cos ^{2} \nu\right) \cos ^{2} \theta+\left(28-24 \cos ^{2} \nu\right) \cos ^{3} \theta \\
& -16 \cos \nu \cos ^{4} \theta+6(3+\cos \nu) \sin \nu \sin \theta \\
& +4(7-3 \cos \nu) \sin \nu \sin \theta \cos \theta-24 \sin \nu \cos \nu \sin \theta \cos ^{2} \theta \\
& -16 \sin \nu \sin \theta \cos ^{3} \theta
\end{aligned}
$$

and

$$
\begin{aligned}
& {[1-\cos (\nu-\theta)]^{2}(1-\cos \theta)^{-1}\left(2 a_{3}+a_{2}-a_{0}\right)} \\
& =-31-16 \cos \nu+9 \cos ^{2} \nu+\left(-29+38 \cos \nu+39 \cos ^{2} \nu\right) \cos \theta \\
& \quad+\left(8+26 \cos \nu-16 \cos ^{2} \nu\right) \cos ^{2} \theta \\
& \quad+\left(36-16 \cos \nu-48 \cos ^{2} \nu\right) \cos ^{3} \theta+6(5+2 \cos \nu) \sin \nu \sin \theta \\
& \quad+2(19-8 \cos \nu) \sin \nu \sin \theta \cos \theta-16(1+3 \cos \nu) \sin \nu \sin \theta \cos ^{2} \theta .
\end{aligned}
$$

After substituting $\theta=2 \arctan (\sin \nu /(d+\cos \nu))$ into the last two expressions, we can see that it suffices to prove that the following two expressions are positive: 


$$
\begin{aligned}
\sum_{k=0}^{8} & q_{k} d^{k} \equiv-2 d^{8}+(15-\cos \nu) d^{7}-\left(46-29 \cos \nu-19 \cos ^{2} \nu\right) d^{6} \\
& +\left(65-131 \cos \nu-46 \cos ^{2} \nu+38 \cos ^{3} \nu\right) d^{5} \\
& -\left(32-215 \cos \nu+71 \cos ^{2} \nu+150 \cos ^{3} \nu-28 \cos ^{4} \nu\right) d^{4} \\
& -\left(11+123 \cos \nu-264 \cos ^{2} \nu-128 \cos ^{3} \nu+128 \cos ^{4} \nu-8 \cos ^{5} \nu\right) d^{3} \\
& +\left(10-9 \cos \nu-183 \cos ^{2} \nu+104 \cos ^{3} \nu+172 \cos ^{4} \nu-40 \cos ^{5} \nu\right) d^{2} \\
& +\left(3+23 \cos \nu+6 \cos ^{2} \nu-134 \cos ^{3} \nu-40 \cos ^{4} \nu+64 \cos ^{5} \nu\right) d \\
& -2-3 \cos \nu+11 \cos ^{2} \nu+14 \cos ^{3} \nu-32 \cos ^{4} \nu-32 \cos ^{5} \nu, \quad(1.24)
\end{aligned}
$$

and

$$
\begin{aligned}
\sum_{k=0}^{6} r_{k} d^{k} \equiv & -8 d^{6}+(52+4 \cos \nu) d^{5}-\left(119-28 \cos \nu-27 \cos ^{2} \nu\right) d^{4} \\
& +\left(92-172 \cos \nu-76 \cos ^{2} \nu+28 \cos ^{3} \nu\right) d^{3} \\
& +\left(18+232 \cos \nu-14 \cos ^{2} \nu-96 \cos ^{3} \nu+12 \cos ^{4} \nu\right) d^{2} \\
& -\left(24+32 \cos \nu-148 \cos ^{2} \nu-60 \cos ^{3} \nu+48 \cos ^{4} \nu\right) d \\
& -15-60 \cos \nu-61 \cos ^{2} \nu+40 \cos ^{3} \nu+48 \cos ^{4} \nu
\end{aligned}
$$

In order to do this, let us partition the ranges for both $d$ and $\nu$ into several parts and find the relevant estimates separately. Assuming that $\nu_{1} \leq \nu \leq \nu_{2}, d_{1} \leq d \leq d_{2}$, we can easily notice that

$$
q_{8} d^{8}+q_{7} d^{7} \geq\left(15-2 d_{2}-\cos \nu\right) d^{7} \geq\left(15-2 d_{2}-\cos \nu\right) d_{1} d^{6},
$$

the first factor being positive. Similarly, it follows that

$$
\begin{aligned}
& q_{8} d^{8}+q_{7} d^{7}+q_{6} d^{6} \\
& \quad \geq\left[15 d_{1}-2 d_{1} d_{2}-46+\left(29-d_{1}\right) \cos \nu+19 \cos ^{2} \nu\right] d_{1} d^{5}
\end{aligned}
$$

However, in this case, in order to prove that the factor is positive we need to make the necessary evaluations for each of the following cases separately.

As a result, we can write

$$
q_{8} d^{8}+q_{7} d^{7}+q_{6} d^{6}+q_{5} d^{5} \geq\left\{\begin{array}{lll}
M_{1} d_{1}^{5} & \text { if } M_{1} \geq 0 \\
M_{1} d_{2}^{5} & \text { if } & M_{1}<0
\end{array}\right.
$$

where

$$
\begin{gathered}
M_{1}=15 d_{1}^{2}-2 d_{1}^{2} d_{2}-46 d_{1}+\left(29 d_{1}-d_{1}^{2}-131\right) \cos \nu_{1} \\
+\left(19 d_{1}-46\right) \cos ^{2} \nu_{2}+38 \cos ^{3} \nu_{2}
\end{gathered}
$$


In a similar way,

$$
q_{4} d^{4} \geq Q_{2}^{\prime} d_{1}^{4}, \quad \text { if } \quad M_{2} \geq 0,
$$

where

$$
M_{2}=-32+215 \cos \nu_{2}-71 \cos ^{2} \nu_{1}-150 \cos ^{3} \nu_{1}+28 \cos ^{4} \nu_{2},
$$

otherwise we shall use rather a crude estimate

$$
\begin{aligned}
q_{4} d^{4} \geq\left(215 \cos \nu_{2}+28 \cos ^{4} \nu_{2}\right) d_{1}^{4} \\
-\left(32+71 \cos ^{2} \nu_{1}+150 \cos ^{3} \nu_{1}\right) d_{2}^{4} .
\end{aligned}
$$

Since the polynomial

$$
g(x)=-11-123 x+264 x^{2}+128 x^{3}-128 x^{4}+8 x^{5}
$$

is positive and increases with respect to $x$ on the whole segment $0.5707 \cdots \leq$ $x \leq 1$, we get

$$
\begin{aligned}
q_{3} d^{3} \geq\left(-11-123 \cos \nu_{2}+\right. & 264 \cos ^{2} \nu_{2}+128 \cos ^{3} \nu_{2} \\
- & \left.128 \cos ^{4} \nu_{2}+8 \cos ^{5} \nu_{2}\right) d_{1}^{3} .
\end{aligned}
$$

Next, we note that

$$
q_{2} d^{2} \geq M_{3} d_{1}^{2}, \quad \text { if } \quad M_{3} \geq 0
$$

where

$$
\begin{aligned}
M_{3}=10-9 \cos \nu_{1}-183 \cos ^{2} \nu_{1}+104 \cos ^{3} \nu_{2} \\
+172 \cos ^{4} \nu_{2}-40 \cos ^{5} \nu_{1},
\end{aligned}
$$

otherwise take

$$
\begin{aligned}
q_{2} d^{2} \geq(10+ & \left.104 \cos ^{3} \nu_{2}+172 \cos ^{4} \nu_{2}\right) d_{1}^{2} \\
& -\left(9 \cos \nu_{1}+183 \cos ^{2} \nu_{1}+40 \cos ^{5} \nu_{1}\right) d_{2}^{2} .
\end{aligned}
$$

Since the polynomial

$$
h(x)=3+23 x+6 x^{2}-134 x^{3}-40 x^{4}+64 x^{5}
$$


is negative and decreases with respect to $x$ for all $0.5707 \cdots \leq x \leq 1$, we get

$$
\begin{aligned}
q_{1} d \geq\left(3+23 \cos \nu_{1}+6 \cos ^{2} \nu_{1}\right. & -134 \cos ^{3} \nu_{1} \\
& \left.-40 \cos ^{4} \nu_{1}+64 \cos ^{5} \nu_{1}\right) d_{2} .
\end{aligned}
$$

Finally, we have

$$
\begin{aligned}
q_{0} \geq-2-3 \cos \nu_{1}+11 \cos ^{2} \nu_{1}+ & 14 \cos ^{3} \nu_{1} \\
& -32 \cos ^{4} \nu_{1}-32 \cos ^{5} \nu_{1} .
\end{aligned}
$$

The polynomial expression in (1.25) is estimated quite analogously. Thus, we have

$$
r_{6} d^{6}+r_{5} d^{5}+r_{4} d^{4} \geq \begin{cases}M_{4} d_{1}^{4} & \text { if } M_{4} \geq 0 \\ M_{4} d_{2}^{4} & \text { if } \quad M_{4}<0\end{cases}
$$

where

$$
M_{4}=52 d_{1}-8 d_{1} d_{2}-119+\left(28+4 d_{1}\right) \cos \nu_{2}+27 \cos ^{2} \nu_{2} .
$$

Furthermore,

$$
r_{3} d^{3}+r_{2} d^{2} \geq\left\{\begin{array}{lll}
M_{5} d_{1}^{2} & \text { if } \quad M_{5} \geq 0 \\
M_{5} d_{2}^{2} & \text { if } \quad M_{5}<0
\end{array}\right.
$$

where

$$
\begin{aligned}
M_{5}=92 d_{2} & +18+\left(232-172 d_{2}\right) \cos \nu_{1}-\left(76 d_{2}+14\right) \cos ^{2} \nu_{1} \\
& +24\left(d_{2}-4\right) \cos ^{3} \nu_{1}+4 d_{2} \cos ^{3} \nu_{2}+12 \cos ^{4} \nu_{2} .
\end{aligned}
$$

The polynomial

$$
\phi(x)=-24-32 x+148 x^{2}+60 x^{3}-48 x^{4}
$$

is positive and increases on the segment $0.5707 \cdots \leq x \leq 1$ and hence, we have

$$
r_{1} d \geq\left(-24-32 \cos \nu_{2}+148 \cos ^{2} \nu_{2}+60 \cos ^{3} \nu_{2}-48 \cos ^{4} \nu_{2}\right) d_{1} .
$$

As the relevant expression is not monotonic with respect to $\nu$ on the whole interval, let us take

$$
r_{0} \geq-15-60 \cos \nu_{1}-61 \cos ^{2} \nu_{1}+40 \cos ^{3} \nu_{2}+48 \cos ^{4} \nu_{2} .
$$


Now, we show how the requested estimates are verified. In the first case, assume that $\nu_{1}=0$ and $\nu_{2}=0.3$. For $d=3.91$ solve the algebraic equation $p(d, x)=0$, the function $p(d, x)$ being defined in (1.23). Since it has only one positive root $x=1.0034 \ldots$, we deduce that $p(3.91, \cos \nu)>0$ for all $\nu$.

On the other hand, by setting in this equation $d=3.81$ we find that its positive root $x=0.9529 \ldots$ is less than $\cos 0.3=0.9553 \ldots$, so that $p(3.81, \cos \nu)<0$ for all $\nu \in[0,0.3]$.

Therefore, if $0 \leq \nu \leq 0.3$, then the solution of (1.5) corresponds to some $d_{\nu}$ such that $3.81 \leq d_{\nu} \leq 3.91$.

In view of estimates (1.26)-(1.42), we conclude that $Q_{+} \geq 8704.9$, and $R_{+} \geq 360.0$, where $Q_{+}, R_{+}$are lower bounds for the expressions in (1.24) and (1.25), respectively.

Table 1 includes estimates obtained in each of the remaining cases. We see that both required expressions are positive, and therefore, the proof of the Theorem 1.3 is complete.

Note that the increment of $\nu$ should be kept steadily decreasing because $\cos \nu$ changes more rapidly for larger values of $\nu$ and so does the difference between the bounds of $d$.

Table 1.

\begin{tabular}{|c|c|c|c|c|c|c|c|}
\hline$\nu_{1}$ & 0.3 & 0.43 & 0.52 & 0.59 & 0.65 & 0.7 & 0.74 \\
\hline$\nu_{2}$ & 0.43 & 0.52 & 0.59 & 0.65 & 0.7 & 0.74 & 0.78 \\
\hline$d_{1}$ & 3.72 & 3.63 & 3.56 & 3.49 & 3.42 & 3.37 & 3.31 \\
\hline$d_{2}$ & 3.82 & 3.73 & 3.64 & 3.57 & 3.5 & 3.43 & 3.375 \\
\hline$Q_{+}$ & 4418.1 & 2507.4 & 5128.4 & 3414.6 & 2278.8 & 2070.6 & 1073.7 \\
\hline$R_{+}$ & 103.6 & 24.5 & 114.5 & 38.1 & 5.8 & 103.1 & 15.0 \\
\hline
\end{tabular}

\begin{tabular}{|c|c|c|c|c|c|c|c|c|}
\hline$\nu_{1}$ & 0.78 & 0.81 & 0.84 & 0.87 & 0.89 & 0.91 & 0.93 & 0.95 \\
\hline$\nu_{2}$ & 0.81 & 0.84 & 0.87 & 0.89 & 0.91 & 0.93 & 0.95 & 0.964 \\
\hline$d_{1}$ & 3.26 & 3.219 & 3.169 & 3.13 & 3.09 & 3.06 & 3.026 & 3 \\
\hline$d_{2}$ & 3.32 & 3.27 & 3.221 & 3.171 & 3.14 & 3.105 & 3.063 & 3.03 \\
\hline$Q_{+}$ & 996.9 & 667.9 & 243.2 & 501.6 & 166.9 & 62.6 & 6.0 & 159.6 \\
\hline$R_{+}$ & 53.8 & 48.1 & 9.7 & 73.6 & 19.1 & 5.7 & 3.1 & 33.1 \\
\hline
\end{tabular}

